\title{
Criterios \\ Métodos de diagnóstico para revestimientos de edificios antiguos. Importancia y aplicabilidad de los ensayos in situ
}

Martha Tavares' $>$ Ana Cristian Magalhães" $>$ Maria do Rosário Veiga"ll $>$ José Aguiar ${ }^{\text {IV }}$

\section{Resumen}

En algunos casos los sentidos humanos pueden detectar los problemas patológicos que afectan a los revestimientos de paredes, pero el uso exclusivo de estos recursos puede revelar datos dudosos o no suficientemente exactos. Así pues, se hace necesario utilizar técnicas auxiliares que completen los sentidos humanos naturales para concluir el diagnóstico del problema.

El uso de los métodos de análisis in situ para revestimientos, como técnicas auxiliares, ha dado resultados interesantes respecto a algunos valores de propiedad, que pueden ser muy útiles en la estimación de su estado de conservación.

Este trabajo tiene como principales objetivos: evidenciar la importancia de algunas técnicas in situ como métodos auxiliares de diagnóstico en revestimientos y evaluar su aplicabilidad y las posibilidades del análisis combinado de los resultados obtenidos con dichas técnicas.

\section{Palabras clave}

\section{Deterioro}

Diagnóstico

Análisis de materiales

Conservación

Restauración

Metodología

Muros

Arquitectura

Ensayo in situ

Técnica no destructiva

Edificios históricos

\section{Introducción}

El problema del mantenimiento de las fachadas externas de un edificio histórico pasa por la conservación de técnicas constructivas tradicionales y por el uso de materiales compatibles y, generalmente, lo más similares posibles a los originales.

Como estos acabados son renovados a lo largo del tiempo, muchos de ellos han sido tratados sin ninguna preocupación de análisis crítico en cuanto a sus valores estéticos, culturales y técnicos. De esta forma, muchos de estos acabados y técnicas ancestrales se han perdido a lo largo del tiempo. En la gran parte de los casos, los revestimientos todavía existentes se encuentran bastante deteriorados, necesitando urgentemente ser restaurados a través de técnicas actuales y, dentro de los conceptos generales de conservación, aplicando la metodología más idónea. Es importante tener en cuenta que los métodos empleados para la conservación y la restauración de revestimientos exteriores varían conforme al tipo y grado de deterioración (patología), y de acuerdo a los materiales necesarios para su preservación.

Por lo tanto, antes de iniciar el tratamiento de restauro, hace falta un estudio histórico y técnico del edificio, una caracterización de las diversas capas de revestimientos, además de un conocimiento exhaustivo de las causas y formas de degradación (TAVARES et al., 2003).

Tal como se sabe, estos estudios todavía carecen de investigación científica, y es por este motivo que el Laboratorio Nacional de Engenharia Civil (LNEC, en Portugal), preocupado por esta temática, viene desarrollando estudios sobre la aplicación de un conjunto de técnicas de ensayos in situ (no-destructivas o poco destructivas) con el objetivo de establecer una metodología general de evaluación del estado de conservación y de diagnóstico de lesiones de revestimientos antiguos ${ }^{1}$.

\section{Los diversos métodos de diagnóstico in} situ para el reconocimiento del estado de conservación de revestimientos antiguos

Los objetivos principales de la aplicación de los métodos de ensayo in situ son:

> Determinar los materiales utilizados y las técnicas de aplicación utilizadas

> Diagnosticar las alteraciones y sus causas

> Seleccionar los métodos de restauración más adecuados

Hoy en día, estos métodos son un complemento fundamental para las disciplinas de la historia del arte y de la restauración, 


\section{Criterios \\ Métodos de diagnóstico para revestimientos de edificios antiguos. \\ Importancia y aplicabilidad \\ de los ensayos in situ \\ Martha Tavares \\ y otros}

contribuyendo a un conocimiento más profundo de la técnica, de los materiales utilizados, de su composición, de su estado de conservación y de su nivel de desempeño.

\section{Observaciones y registros: investigación histórica y técnica sobre el cromatismo del edificio a lo largo de los años}

\section{Investigación histórica}

Esta investigación debe permitir conocer el proceso histórico, desde la época de la construcción hasta el momento de la intervención, permitiendo situar el edificio en su contexto histórico, conocer su edad y las principales transformaciones que sufrió, suministrando datos acerca de los materiales y técnicas utilizados.

Deben identificarse las lesiones existentes en el edificio, fundamentalmente en los revestimientos, y su grado de riesgo, a través de fichas de obra y registros fotográficos y gráficos. Se estudian las condicionantes externas, como orientación, vientos predominantes, Iluvia, valores medios y extremos de humedades relativas y temperaturas, para predecir de qué modo afectan los materiales constructivos y cómo contribuyen en el proceso de deterioro.

Se identifican también los tipos de revestimientos presentes en el edificio, en cuanto a su técnica, composición, textura y color, a través de registros escritos, fotografias y fichas de obra. Para la realización de esta inspección, los técnicos pueden recurrir, además de a la observación a ojo desnudo, a diversas técnicas auxiliares.

\section{Registro fotográfico}

La fotografía con radiación visible es importante para registrar el estado de conservación de la obra. Pueden ser utilizadas varias técnicas fotográficas:

\section{$>$ Fotografías simples y macro}

> Fotografía con iluminación tangencial o rasante, con ángulos de $5^{\circ}$ a $30^{\circ}$, permitiendo observar el estado general y las irregularidades de la superficie (falta de verticalidad, abombamientos, etc.).

$>$ Fotogrametría. Esta metodología constituye un instrumento muy eficiente para el levantamiento arquitectónico en la fase preliminar de restauración. La fotogrametría estudia el sistema de proporciones y dimensiones de un objeto a partir de registros fotográficos en película o digitales, permitiendo obtener levantamientos planimétricos de objetos reales fotografiados anteriormente, además de informaciones pertinentes sobre su morfología y sus elementos estructurales y arquitectónicos. Así se permite definir y reconstituir con precisión la forma, dimensión y posición en el espacio del objeto.
' Restauradora. Becaria de investigación en el Laboratório Nacional de Engenharia Civil (LNEC). Lisboa, Portugal

" Ingeniera Civil. Becaria de investigación en el Laboratório Nacional de Engenharia Civil (LNEC). Lisboa, Portugal

III Ingeniera Civil. Investigadora en el Laboratório Nacional de Engenharia Civil (LNEC). Lisboa, Portugal

IV Arquitecto. Investigador en el Laboratório Nacional de Engenharia Civil (LNEC). Lisboa, Portugal

La fotografía con radiación invisible es utilizada en el estudio de la patología de la obra y pueden utilizarse diversas técnicas:

$>$ Fotografía de ultravioleta, que permite determinar algunos tipos de alteración de las superficies e identificar repinturas, así como observar las condiciones de algunos barnices y de otros materiales.

$>$ Fotografía de infrarrojo, con la que se consigue observar detalles como los "arrepentimientos"2 del autor y repinturas.

> Reflectografía de infrarrojo, que posibilita la observación de los dibujos subyacentes, asignaturas, "arrepentimientos" y reconocer algunos pigmentos.

$>$ Radiografía con rayo $x$, con la que se pueden identificar alteraciones, intervenciones anteriores y superposición de distintas capas.

\section{Investigación cromática}

Esta investigación implica la elección previa de los locales que previsiblemente hayan sufrido pocas modificaciones y comprende:

> La realización de una investigación histórica sobre el cromatismo del edificio a lo largo del tiempo a través de prospecciones arqueológicas, con remoción mecánica (a través de bisturí) de las diversas camadas de pinturas existentes.

> La recogida de muestras del revoco con información relevante sobre los diversos sistemas de revestimiento y pintura sobrepuestos para la realización del análisis de laboratorio.

$>$ La identificación de los colores encontrados a través de un análisis comparativo con determinados padrones, como los Atlas Cromáticos Munsell o NCS (Natural Colour Sistems). En caso en que fuera necesario y justificable, la lectura cromática de los colores puede ser realizada a través de espectrocolorimetro, y sus resultados traducidos en los sistemas $\mathrm{CIE}(X, Y, Z)$ y CIELAB.

Los resultados obtenidos con estas investigaciones, después de analizados críticamente y en función de su interpretación estética, histórica, pero además material (química y física), pueden fundamentar con más seguridad la definición de un concepto de presentación de las superficies y su consecuente esquema, tabla o plano de color.

\section{Métodos de ensayos in situ: evaluación del estado de conservación del revestimiento}

Los métodos de ensayo in situ para evaluar el estado de conservación de los morteros antiguos y su comportamiento pueden ser clasificados en no-destructivos, semi destructivos y destructivos, 

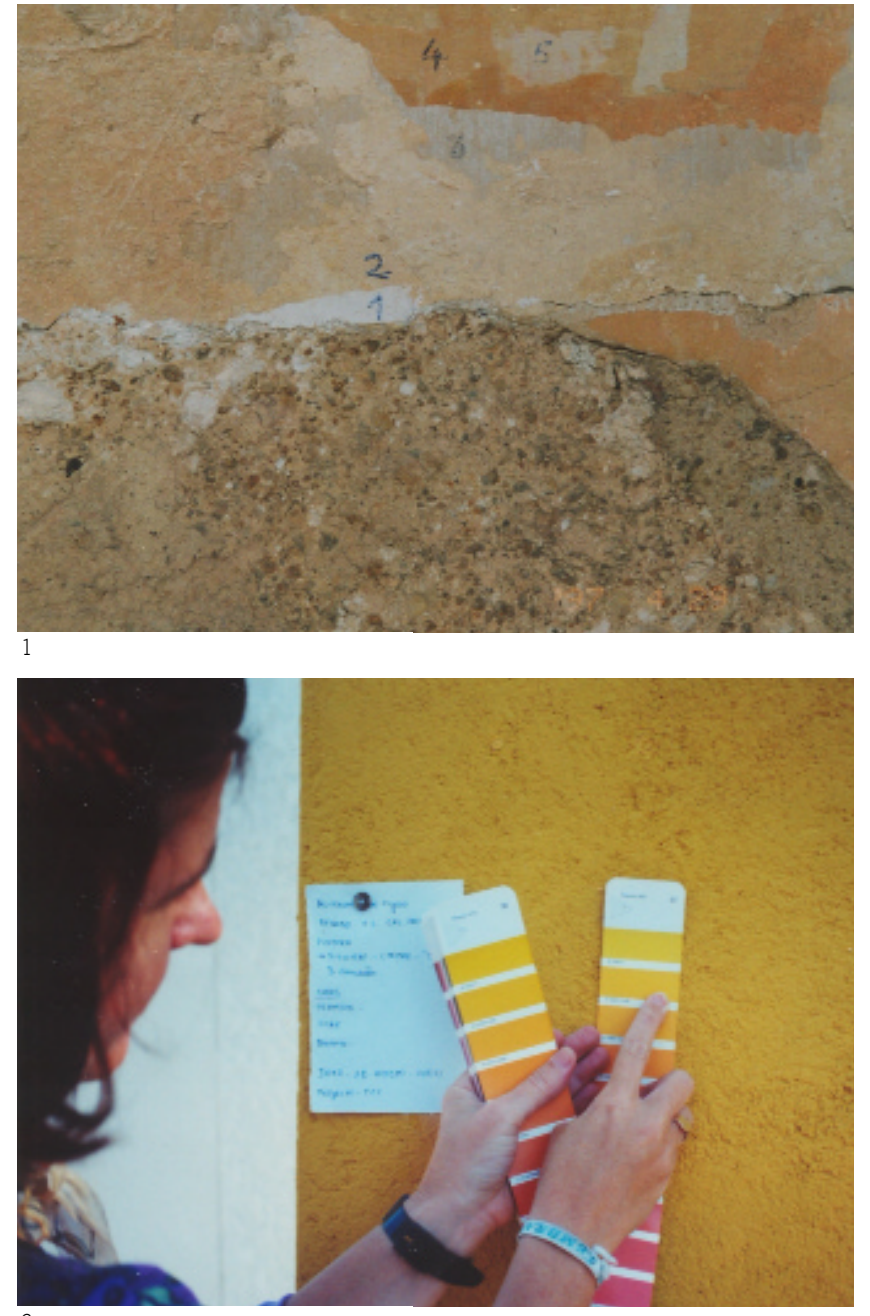

en base al grado de intrusión que producen en el revestimiento que vendrá a ser estudiado.

\section{a) Ensayos no-destructivos}

Los métodos de ensayo no-destructivos tienen gran importancia en el estudio de materiales de construcción porque permiten la determinación de sus características sin deteriorarlos. Consisten esencialmente en procedimientos que se basan en el análisis del comportamiento de una determinada propiedad física en el interior o en la superficie de un material.

Entre las diversas técnicas de ensayo no-destructivos, se resumen en este trabajo las que se consideran más propicias para la caracterización del comportamiento de revestimientos de paredes.

> Ensayo de permeabilidad al agua a baja presión (con los Tubos de Karsten)
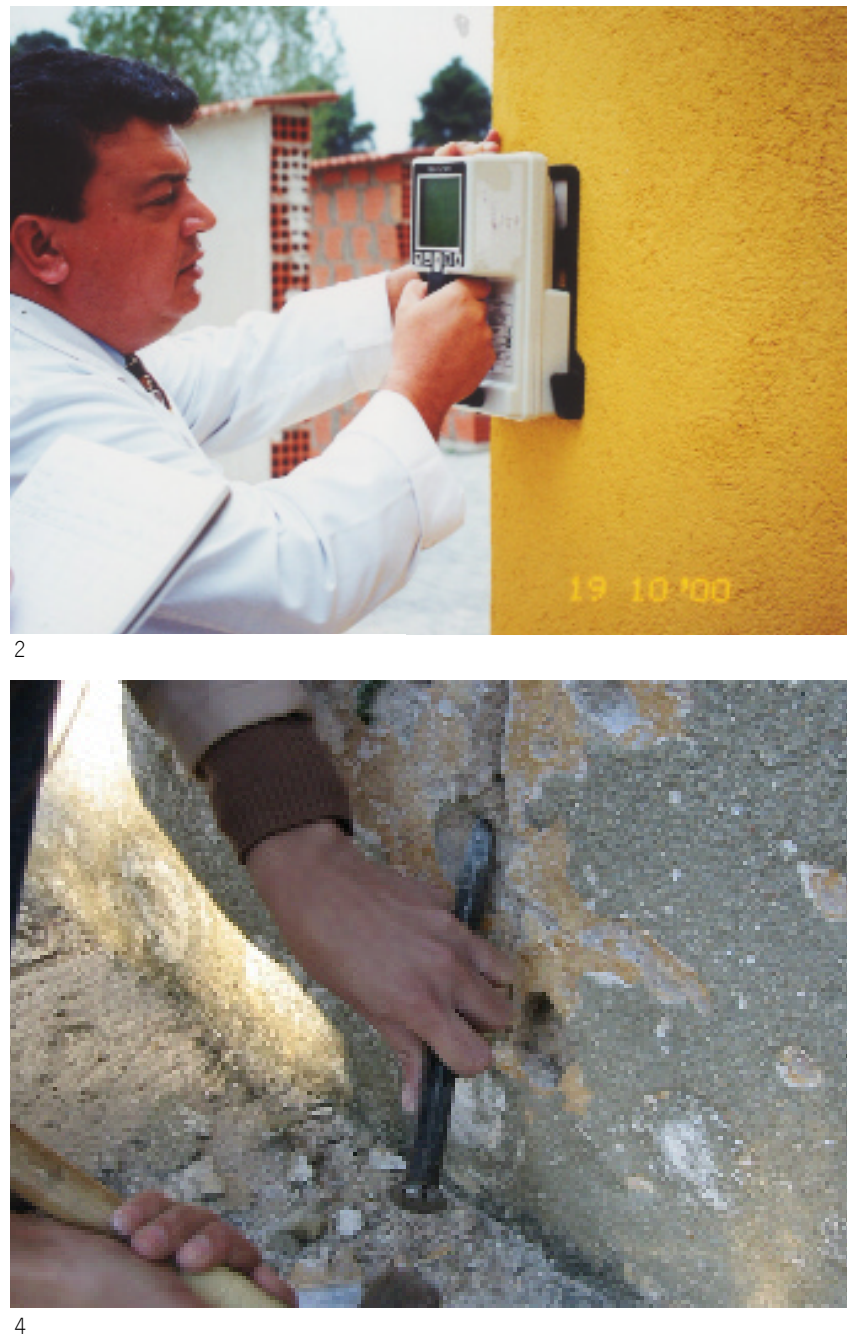

La técnica de ensayo, basada en una metodología adoptada por RILEM (RILEM, 1980), consiste en medir la cantidad de agua absorbida por una determinada superficie del revoco o de acabado, después de transcurrido un determinado tiempo. Se utiliza para esto un conjunto de pequeños tubos graduados y en forma de pipa, que son fijados a las zonas de la pared que se pretende estudiar.

La medida de la permeabilidad al agua a través de este método puede ser usada para caracterizar, cualitativamente, el estado en que se encuentra el revoco. En efecto, a través de comparaciones que tienen por objeto evaluar las alteraciones superficiales que modifican la absorción del agua por el revoco es posible estimar su grado de degradación.

\section{$>$ Ultrasonidos}

Es una técnica totalmente no destructiva, de empleo bastante corriente en la evaluación de las características de varios tipos de 


\begin{tabular}{|l|}
\hline Criterios \\
Métodos de diagnóstico \\
para revestimientos de \\
edificios antiguos. \\
Importancia y aplicabilidad \\
de los ensayos in situ \\
Martha Tavares \\
y otros
\end{tabular}
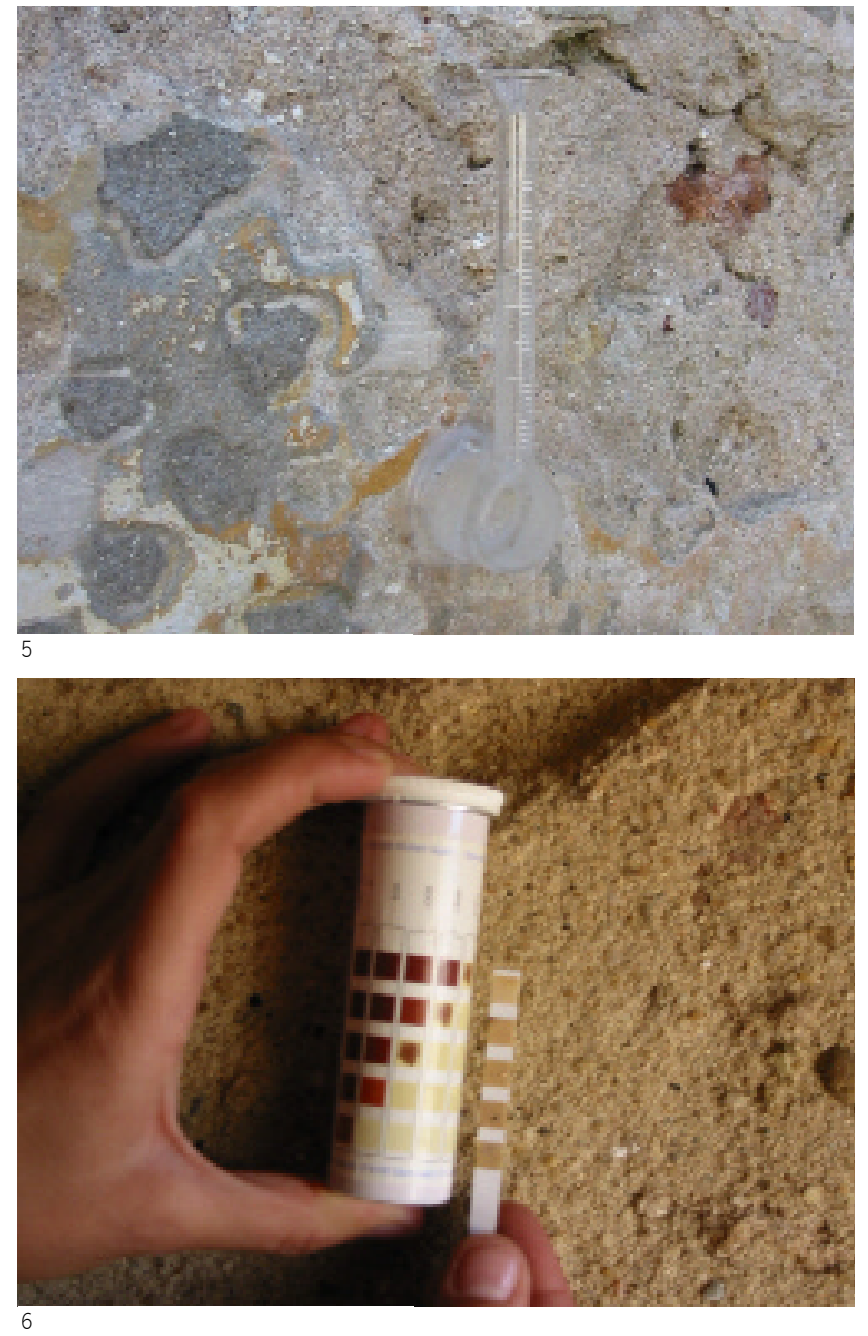

materiales de construcción en general. En edificios, las aplicaciones son variadas: estudio de la homogeneidad de materiales, comparación de determinadas características como la resistencia y el módulo de elasticidad, estimativa de alteraciones cualitativas del material a lo largo del tiempo, etc.

Dicha técnica se basa en la emisión de vibraciones acústicas de la misma naturaleza que el sonido, pero con frecuencia superior que, al atravesar los materiales, permiten detectar alteraciones significativas en sus características, a través de la variación de su velocidad de transmisión. En combinación con otros métodos de ensayo no-destructivos, permite mejorar la exactitud de las estimativas de resistencia de los materiales analizados. Algunos ensayos preliminares con esta técnica fueron realizados en el ámbito de un Proyecto de investigación en curso en el LNEC (MAGALHÃES et al., 2003 y PINA et al., 2003).
$>$ Termografía de infrarrojos

Ésta es una técnica que tiene relevante interés entre los métodos nodestructivos de diagnóstico de las lesiones de los revestimientos, pudiendo ser utilizada para evaluar su estado de conservación -la distribución de la humedad en éste, la existencia de zonas con falta de adherencia, no continuidades de la superficie, fisuras, etc.- y también en la evaluación de la compatibilidad de revestimientos de sustitución.

La técnica se basa en los análisis de las imágenes obtenidas para permitir relacionar las heterogeneidades térmicas con algunos factores de degradación, y con las características de los materiales.

El hecho de ser una técnica de utilización rápida y de que el aparato sea ligero y de pequeñas dimensiones, permitiendo su uso en locales de difícil acceso, confieren alguna practicidad a este método al tratarse de un medio de diagnóstico in situ. Investigaciones recientes fueron o están siendo realizadas con este fin (MOROPOULO et al., 2000; PINA et al., 2002, PINA et al., 2003).

\section{$>$ Endoscopia}

Es una técnica óptica que recurre a endoscopios, lo que torna posible inspeccionar áreas que no son directamente observables a ojo desnudo. En edificios antiguos, este método tiene diversas aplicaciones: detección de degradaciones en el interior de la albañilería, inspección de forjados a través de techos falsos, inspección de pavimentos, comprobación del estado interior de las conductas o del interior del techo de una cubierta, detección de fisuras interiores, etc.

\section{> Humidimetro portátil}

Es un instrumento bastante simple, utilizado para medir el contenido de agua en las paredes a través de dos electrodos en forma de agujas o de sondas de profundidad que son introducidos en los elementos a analizar (MASSARI, 1993; HENRIQUES, 2001). A pesar de la poca precisión de la técnica y de la interpretación de los resultados, que está vinculada al uso de tablas convertidotas $^{3}$, esta técnica, utilizada en términos comparativos y no absolutos, puede ser una herramienta bastante útil en la detección de zonas con elevado contenido de humedad y en la determinación de las causas de dicho fenómeno.

> Identificación y determinación del contenido de sales solubles El análisis semi cuantitativo de las sales solubles puede ser realizado a través de métodos rápidos, poco onerosos y bastante eficaces. Existen en el mercado equipamientos portátiles que permiten verificar la existencia y el tipo de sales solubles sobre la superficie de las paredes de una forma simple y rápida (BORELLI, 1999). Estos aparatos objetivan, por lo tanto, una evaluación preliminar de la eventualidad de ocurrencia de lesiones debidas a fenómenos de higroscopicidad, o la confirmación de resultados de aparatos usados para determinación del contenido de agua, extremamente sensible a la existencia de las sales solubles. 
5. Ensayo de permeabilidad con Tubos de Karsten

6. Ensayo strip para identificación de las sales

7. Ensayo de choque de esfera

8. Ensayo de penetración controlada

Los ensayos se basan en la identificación de reacciones químicas características de ciertas sales. Los ensayos microquímicos para identificación de las sales son ensayos relativamente simples de reacciones químicas que pueden identificar el tipo de aniones (iones negativos derivados de la disolución de las sales: sulfatos, cloruros, nitratos) presentes en la solución y, aunque no ofrezcan una información cuantitativa exacta, son capaces de distinguir la masa cuantitativa de los diferentes aniones, dando una indicación preliminar de la degradación observada; el ensayo strip sirve para identificar un ion específico. Se recurre a marcadores impregnados de determinados compuestos que, inmersos en una solución, varian la intensidad del color, conforme la cantidad de iones presentes en la superficie del material a ensayar; los Kits colorimétricos se basan en las reacciones que conducen a la formación de compuestos coloridos, formados por cada ion. La intensidad del color indica la cantidad de iones presentes en la solución. Los Kits existentes en el mercado contienen todos los reactivos y materiales necesarios para el análisis.

\section{b) Ensayos destructivos y poco destructivos}

El uso de ensayos poco destructivos y destructivos, teniendo en consideración la posibilidad de interpretación de los resultados en conjunto, constituye también un adecuado medio auxiliar de diagnóstico para los revestimientos, además de un instrumento para definición de medidas a tomar en posibles intervenciones. No obstante, dichos ensayos deben ser tan poco intrusivos como sea posible, para evitar la destrucción excesiva de los elementos que se procura preservar, por lo que siempre que se pueda, debemos privilegiar el recurso a ensayos in situ no destructivos (VEIGA et al., 2001).

$>$ Ensayos de resistencia superficial: impacto de esfera e impacto con elemento cortante

El ensayo de impacto de esfera consiste en la aplicación del impacto de un cuerpo duro de 3 julios de energía, realizado a través de una esfera de acero con cerca de $50 \mathrm{~mm}$ de diámetro. Se obtienen así informaciones sobre la deformación del revestimiento. La resistencia al impacto es evaluada a partir del diámetro de la marca producida en la superficie ensayada y del tipo de degradación resultante (VEIGA et al., 2000; VEIGA et al., 2001).

El ensayo de impacto con elemento cortante es similar a excepción de que, en lugar de la esfera, se utiliza un elemento cortante para producir el impacto. Se obtiene así información sobre la cohesión superficial del material y, eventualmente, sobre la adherencia de la capa superficial. Se hace la evaluación en base al porcentaje de área de revestimiento despegado, en relación al área afectada por el impacto del elemento cortante (VEIGA et al., 2000; VEIGA et al., 2001).

\section{$>$ Ensayo de penetración controlada ${ }^{4}$}

Este ensayo consiste en provocar la penetración de un punzón de acero, con una escala graduada en milímetros, con varios im-
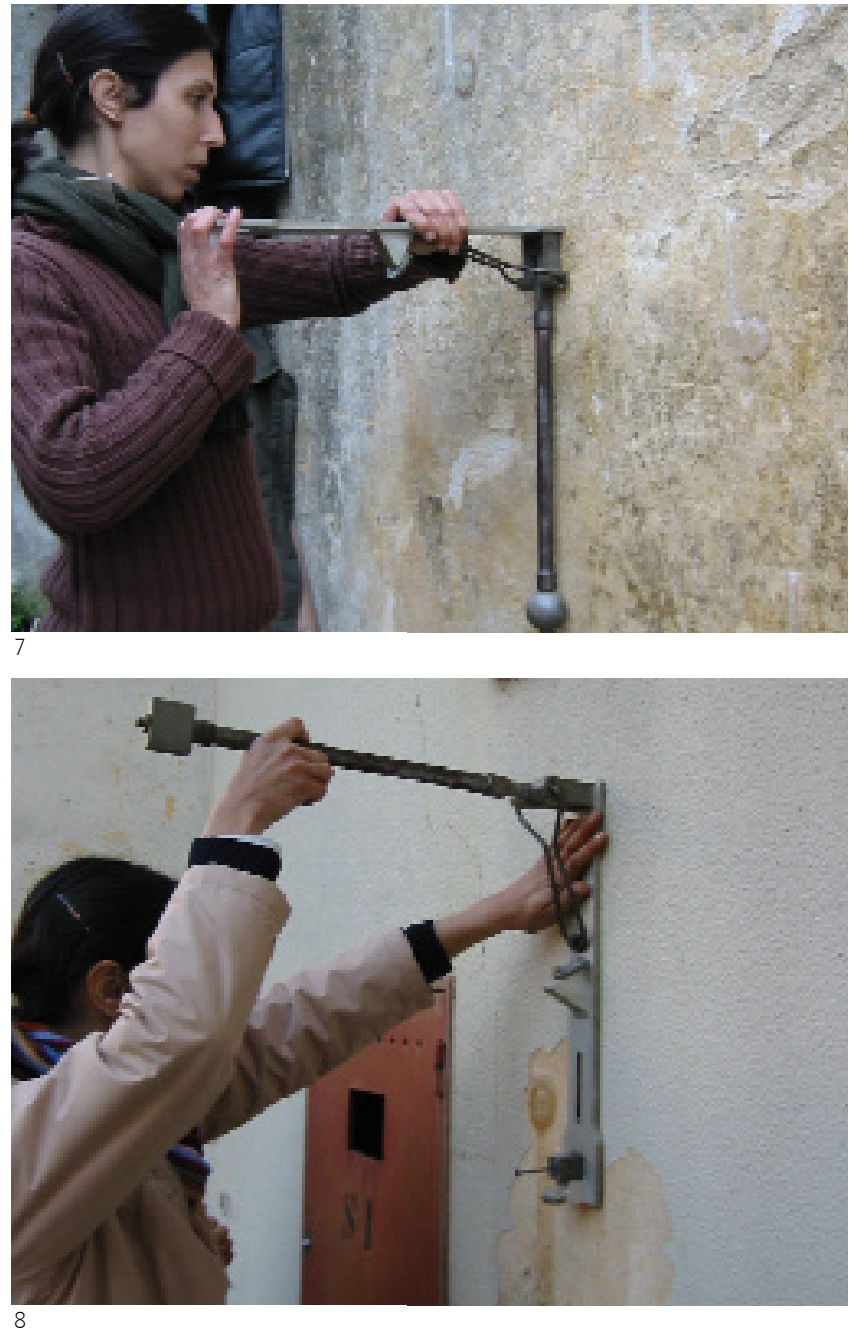

pactos de energía constante. Se puede registrar la penetración obtenida en el mortero con cada impacto efectuado. Este método permite evaluar cualitativamente la resistencia de cada capa y puede ser útil para obtener información sobre la resistencia mecánica de las capas internas, permitiendo de esta forma una evaluación de su estado (VEIGA et al.,2000; VEIGA et al., 2001).

> Ensayos de cohesión (resistencia al rayado y abrasión) Los ensayos de rayado y abrasión son ensayos superficiales, particularmente útiles para capas de acabado de revocos o de estucos, o para superficies pintadas. En ensayos anteriores (VEIGA et al., 2000; MAGALHÃES et al., 2002) también se han revelado adecuados para evaluar la cohesión superficial de revocos y de juntas de asentamiento espesas, verificándose la posibilidad de testar capas más profundas.

El ensayo de rayado es realizado con un disco metálico que raya la superficie a lo largo de $15 \mathrm{~cm}$, con fuerza constante, mientras 


Criterios
Métodos de diagnóstico
para revestimientos de
edificios antiguos.
Importancia y aplicabilidad
de los ensayos in situ
Martha Tavares
y otros

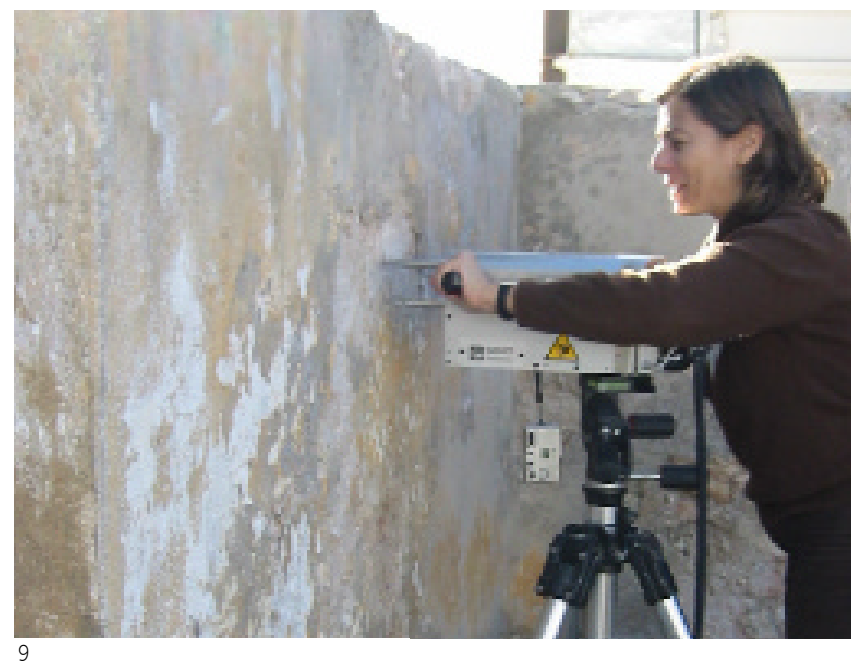

que el de abrasión, adecuado para superficies más friables, utiliza una lija. La evaluación de los resultados de estos ensayos tiene en cuenta la profundidad de la raya producida y la cantidad de material extraído en el rayado.

\section{$>$ Ensayo de adherencia por tracción}

Este ensayo permite evaluar la adherencia del revestimiento al soporte y el modo de rotura previsible. Sin embargo, presenta algunas limitaciones: sólo tiene algún significado en zonas bastante homogéneas y en revestimientos y soportes en relativo buen estado; en caso contrario, la dispersión de resultados y la disgregación del mortero imposibilitan la evaluación. La perforación necesaria que se hace en el revestimiento para fijar el aparato en la pared introduce vibraciones que pueden conducir por sí sola a la pérdida de adherencia o a la disgregación del mortero (VEIGA et al., 2001).

\section{$>$ Ensayo de microperforación}

El ensayo de microperforación pretende evaluar la resistencia mecánica del material cuando ha sido perforado y el resultado obtenido representa su dureza a perforación en profundidad. El ensayo consiste en la producción controlada de un pequeño orificio, durante el cual el equipamiento mide la distancia recorrida y la fuerza de la resistencia ofrecida por el material.

Los valores de la dureza a la perforación medidos a través de dicho método dependen de diversos factores, tales como la composición y el estado de cohesión de los materiales, la velocidad de rotación y taza de penetración utilizada para la perforación, las características de las punteras y la presencia de humedad en los materiales en causa.

Entretanto, es un método cuya aplicación al estudio de morteros es aún experimental, ya que el equipo fue desarrollado originalmente para el ensayo de piedras de durezas variables ${ }^{5} \mathrm{y}$, por lo
9. Ensayo de microperforación

10. Ensayo de adherencia por tracción

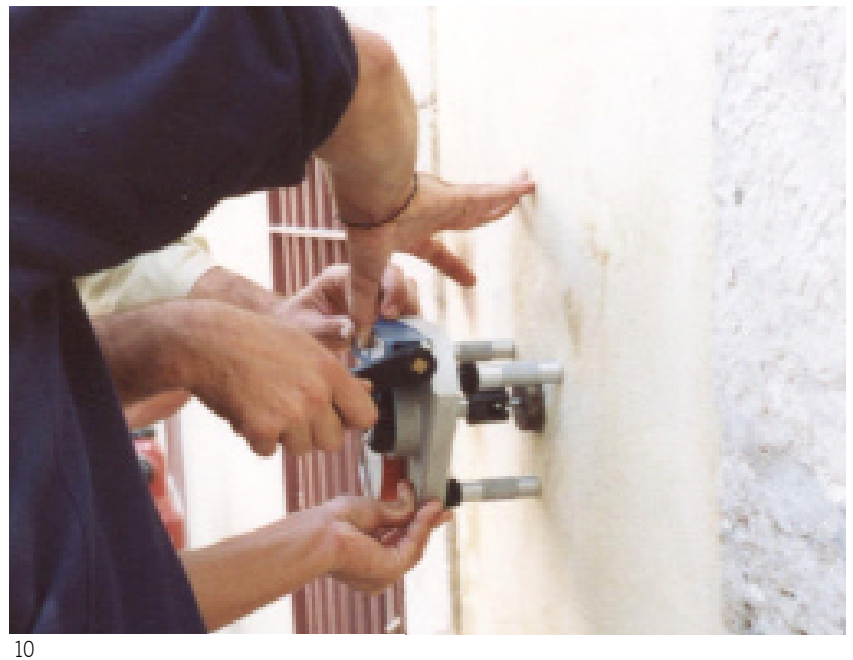

tanto, la adecuación de su uso en revestimientos aún está siendo analizada en el LNEC (MAGALHÃES et al., 2003).

\section{Consideraciones finales}

Los estudios con las técnicas in situ aquí referidas aún se encuentran en desarrollo en el ámbito del Proyecto descrito y en curso, buscando perfeccionar los métodos de ensayos disponibles. Mientras tanto, estos estudios nos proporcionan de forma inmediata elementos sobre la viabilidad del uso de las diversas técnicas. De esta forma, se destacan los siguientes aspectos considerados relevantes durante el estudio:

> Los métodos de evaluación in situ del estado de conservación de los revestimientos antiguos son, en su gran mayoría, ensayos prácticos y poco onerosos. Muchos de los instrumentos usados son ligeros, pequeños y de fácil transporte, permitiendo su uso en locales de difícil acceso, lo que confiere una gran practicidad a estos métodos de ensayos.

$>$ Los métodos no-destructivos son muy importantes para complementar la información de laboratorio, además de permitir la determinación de algunas caracteristicas y lesiones presentes en los revestimientos sin deteriorarlos. No obstante, algunos resultados son escasos y algunos instrumentos tienen limitaciones en cuanto a la viabilidad de realización y de interpretación, necesitando asi de informaciones complementarias.

> La comparación de los datos obtenidos en la investigación histórica con la información recogida con los métodos de evaluación in situ suministra una importante contribución para obtener la historia material del edificio, identificar los elementos que no son originales, caracterizar las lesiones y entender sus causas, tipificar 
las formas de deterioración y establecer de esta forma, una metodología de conservación, restauración o renovación más adecuada.

> Asimismo, en la evaluación de los resultados de las mediciones realizadas in situ hay que tener en cuenta la influencia de diversos parámetros que son imposibles de controlar (por ejemplo, las condiciones climáticas en el momento de la realización de los ensayos, el grado de agua de los materiales en dicho instante, condiciones externas diversas, etc.) y hacer, siempre que sea posible, una evaluación comparativa y no absoluta.

> La aplicación de algunos de los métodos auxiliares aquí referidos (ultrasonidos, termografía de infrarrojos y microperforación) al estudio de los revestimientos y superficies de paredes está, todavía, insuficientemente estudiada, exigiendo nuevas investigaciones que permitan profundizar diversos aspectos, relativos a las condiciones más adecuadas para la ejecución de los ensayos, o relacionados con la interpretación de los resultados obtenidos (PINA et al., 2003, MAGALHÃES et al., 2003).

$>$ El análisis de los resultados de diversos estudios ya realizados (VEIGA et al., 2000, MAGALHÃES et al., 2002) muestra que los ensayos destructivos y poco destructivos referidos -impacto de esfera o impacto con elemento cortante, penetración controlada, cohesión y adherencia por tracción- son, en principio, viables para la evaluación del estado de conservación de revestimientos de paredes antiguas, porque permiten distinguir el comportamiento de varios tipos de revocos y presentan, de un modo general, valores coherentes con los que conocemos las características de los morteros ensayados.

> Algunos ensayos - permeabilidad al agua a baja presión (con los Tubos de Karsten), identificación y determinación de sales, humidímetro portátil y los ensayos mecánicos referidos- se muestran viables, además, en la evaluación de la compatibilidad de revestimientos de sustitución aplicados en edificios antiguos.

\section{Notas}

${ }^{1}$ Dicho trabajo forma parte del proyecto "Metodologias para a Mitigação do Risco Associado à Degradação das Construções", realizado en el LNEC y subvencionado por la Fundação para a Ciência e Tecnologia (FCT). El proyecto de investigación aún se encuentra en curso, por lo que no todos los métodos de ensayos aquí presentados fueron ensayados por los autores.

${ }^{2}$ Modificación de parte de la composición de una pintura por el propio artista durante la fase de ejecución cromática.

${ }^{3}$ Estos aparatos, en general, están calibrados para el uso en determinados tipos de materiales.

${ }^{4}$ Dicho método de ensayo fue desarrollado en el ámbito del proyecto OLDRENDERS

${ }^{5}$ Dicha técnica fue desarrollada recientemente en un proyecto de investigación de la Comunidad Europea - "Development of a new measurement method to determine the superficial hard ness of exposed monumental rocks" - para el ensayo de piedras de durezas variables. Sobre este tema consultar: Tiano et al. Ob. Cit. 2001

\section{Bibliografía}

BORRELLI, E. Conservation of Architectural heritage, historic structures and materials-salts. Roma: ICCROM, 1999

HENRIQUES, F. Humidade em paredes. Lisboa: LNEC, 2001 (2ª edición)

MAGALHÃES, A.C.; VEIGA, M.R.; CARVALHO, F. Diagnosis of anomalies of wall renderings. Experimental techniques for in situ application. XXX IAHS World Congress on Housing. Coimbra: septiembre 2002

MAGALHÃES, A.C.; VEIGA, M.R.; COSTA, D. Diagnóstico de anomalias de revestimientos de paredes con técnicas de ensayo in situ. Avaliação da resistência mecânica. Encontro Sobre Conservação e Reabilitação de Edifícios $3^{\circ}$ ENCORE. Lisboa: LNEC, mayo, 2003

MASSARI, I. Damp Buildings, old and new. Roma: ICCROM, 1993

MOROPOULOU, A.; AVDOIDIS, N.P.; KOUI M. Compatibility assessment of building materials using Infrared Thermography. Proceedings of "15th World Conference on Non destructive Testing". Roma, octubre 2000

PINA SANTOS, C.; MATIAS, L.; MAGALHÃES, A.C.; VEIGA, M.R. Application of thermography and ultra-sounds for wall anomalies diagnosis a laboratory research study. In Proceedings of Non-Destructive Testing in Civil Engineering (NDTCE). Berlin, 2003

PINA SANTOS, C.; MATIAS, L. Application of thermography for moisture detection. A laboratory research study. XXX IAHS World Congress on Housing. Coimbra: septiembre, 2002

RILEM. Water absorption under low pressure. Pipe method. Test $N^{\circ} \| .4$, Tentative Recommendations. París: RILEM, 1980

TAVARES, M.; AGUIAR, J.; VEIGA, M.R. Conservação e restauro de antigos revestimentos exteriores: caracterização de alguns casos do séc. XVII e XIX na zona de Lisboa. V SBTA - Simpósio Brasileiro de Tecnologia das Argamassas. São Paulo: USP, junio 2003

TIANO, P.; DELGADO RODRIGUES, J.; DE WITTE, E.; VERGÈS-BELMIN, V.; MASSEY, S.; SNETHLAGE, R.; COSTA, D.; CADOT-LEROUX, L.; GARROD, E.; SINGER, B. The conservation of monuments: A new method to evaluate consolidating treatments. International Journal for the Restoration of Buildings and Monuments. 2000, vol.6, $n^{\circ} 2$, p.133-150

VEIGA, M.R.; CARVALHO, F. Experimental characterisation of lime based rendering and repointing mortars. Definition of relevant laboratorial and "in situ" tests. Athens: National Technical University of Athens, diciembre 2000

VEIGA, M.R.; AGUIAR, J.; CARVALHO, F.; SANTOS SILVA, A.; SILVA, C. Metodologias para caracterização e conservação de argamassas de revestimento de edificios antigos. Informe Final del Proyecto OLDRENDERS. Lisboa, LNEC, octubre de 2001 